\title{
Reduced hemodynamic load aids low-dose resveratrol in reversing cardiovascular defects in hypertensive rats
}

\begin{abstract}
Sijo Joseph Thandapilly ${ }^{1,2}$, Xavier Lieben Louis ${ }^{1,2}$, John Behbahani ${ }^{3}$, Ali Movahed ${ }^{4}$, Liping Yu ${ }^{1,5}$, Robert Fandrich ${ }^{2}$, Shetuan Zhang ${ }^{6}$, Elissavet Kardami ${ }^{2}$, Hope D Anderson ${ }^{1,3}$ and Thomas Netticadan ${ }^{1,2,5}$

Cardiac hypertrophy and associated myocardial remodeling is one of the main complications of hypertension resulting in the development of heart failure. It is of great significance to explore novel treatments to reverse cardiac hypertrophy in hypertensives with or without affecting blood pressure. In the present study, we investigated whether low-dose resveratrol alone or in a combination with a blood pressure-lowering agent can reverse hypertension-induced cardiovascular dysfunction. Twenty-week-old male spontaneously hypertensive rats (SHRs) and Wistar-Kyoto rats were treated with resveratrol $\left(2.5 \mathrm{mg} \mathrm{kg}^{-1}\right.$ per day) and/or hydralazine ( $25 \mathrm{mg} \mathrm{kg}^{-1}$ per day) for 8 weeks. Blood pressure, cardiac structure and function, and electrocardiogram measurements were examined. Pressure myography of resistance arteries, histological examinations of heart tissues, oxidative stress and inflammatory measurements were also preformed to assess the efficacy of the treatment. Although resveratrol treatment alone was ineffective in reducing systolic blood pressure, diastolic blood pressure, diastolic dysfunction and vascular remodeling, it significantly prevented the systolic impairment and reduced myocardial fibrosis, and reduced oxidative stress and inflammation in hypertensive rats. Furthermore, a combination of resveratrol with hydralazine treatment significantly reduced blood pressure, improved systolic and diastolic function, decreased fibrosis and improved vascular geometry. In summary, low-dose resveratrol itself was unable to reduce systolic blood pressure, diastolic blood pressure, diastolic dysfunction and vascular remodeling. However, resveratrol alone alleviated cardiac fibrosis and some of the functional abnormalities in SHRs. And a combination of resveratrol with hydralazine was more effective than resveratrol or hydralazine alone in improving overall cardiovascular parameters. Hypertension Research (2013) 36, 866-872; doi:10.1038/hr.2013.55; published online 20 June 2013
\end{abstract}

Keywords: cardiovascular; hydralazine; hypertrophy; resveratrol; spontaneously hypertensive rat

\section{INTRODUCTION}

Despite advances in the management and treatment of high blood pressure, cardiovascular complications and associated mortality due to hypertension continue to be on the rise. ${ }^{1}$ Cardiac hypertrophy is one of the main complications of hypertension, which results in the development of heart failure. ${ }^{2}$ Considering the fact that lowering of the hemodynamic load (blood pressure) is an important factor, but not the sole factor, for the treatment of cardiac hypertrophy, ${ }^{3}$ it is important to explore the potential of novel treatments which can regress cardiac hypertrophy in hypertensives with or without affecting blood pressure.

Resveratrol, a polyphenol found predominantly in grapes, has been reported to render strong cardioprotection against various diseases such as obesity, diabetes, hypertension and ischemic heart disease. ${ }^{4-6}$ $\mathrm{We}^{7-9}$ and others ${ }^{10-13}$ have earlier reported that resveratrol was beneficial in preventing cardiac hypertrophy and contractile dysfunction in different models of hypertension. Furthermore, the cardioprotective effect of low dose of resveratrol (close to dietary levels) was reported to be independent of blood pressure-lowering effect, suggesting that resveratrol might act directly on the myocardial tissues. This notion was consistent with our recent study demonstrating the effectiveness of resveratrol in preventing the development of hypertrophy in adult rat cardiomyocytes. ${ }^{14}$

Although, previous studies have examined the potential of resveratrol in preventing cardiac hypertrophy and associated functional abnormalities in different animal models of hypertension, no study has been performed to determine whether resveratrol alone or in a combination with a blood pressure-lowering agent is beneficial in reversing hypertension-induced cardiac hypertrophy and contractile dysfunction. Accordingly, in this study we demonstrate the

${ }^{1}$ Heart Failure Research Laboratory, Canadian Centre for Agri-Food Research in Health and Medicine, St. Boniface Research Centre, Winnipeg, Manitoba, Canada; ${ }^{2}$ Department of Physiology, University of Manitoba, Winnipeg, Manitoba, Canada; ${ }^{3}$ Faculty of Pharmacy, University of Manitoba, Winnipeg, Manitoba, Canada; ${ }^{4}$ Department of Endocrine and Metabolic Diseases, Bushehr University of Medical Sciences, Bushehr, Iran; ${ }^{5}$ Agriculture and Agri-Food Canada, Winnipeg, Manitoba, Canada and ${ }^{6}$ Department of Physiology, Queen's University, Kingston, Ontario, Canada

Correspondence: Dr T Netticadan, Heart Failure Research Laboratory, Canadian Centre for Agri-Food Research in Health and Medicine, St. Boniface Research Centre, Winnipeg, Manitoba, Canada R2H 2A6,

E-mail: tnetticadan@sbrc.ca

Received 27 August 2012; revised 22 February 2013; accepted 22 March 2013; published online 20 June 2013 
effectiveness of resveratrol alone or in combination with hydralazine, a blood pressure-lowering agent in a well-established hypertension model, the spontaneously hypertensive rat (SHR).

\section{MATERIALS AND METHODS}

The experimental protocols used in this project were approved by the University of Manitoba Animal Care Committee and are in agreement with the Canadian Council on Animal Care and Use of Experimental Animals.

\section{Animal model}

Twenty-week-old male SHRs and their controls Wistar-Kyoto (WKY) rats obtained from Charles River, St Constant, Quebec, Canada, were used in this study. Animals were acclimatized for 1 week and maintained in temperature and humidity-controlled rooms with a 12-h dark and 12-h light period cycle.

\section{Treatment and examinations}

Twenty-week-old SHRs and WKY rats were treated with resveratrol and/or hydralazine for 8 weeks. Both resveratrol and hydralazine (Sigma-Aldrich, Oakville, ON, Canada) dissolved in 50\% ethanol (vehicle) were administered daily by oral gavage ( $1 \mathrm{ml}$ per rat) at a dosage of $2.5 \mathrm{mg} \mathrm{kg}^{-1}$ body weight (an effective concentration taken from our previous studies) ${ }^{8,9}$ and $25 \mathrm{mg} \mathrm{kg}^{-1}$ body weight, ${ }^{15}$ respectively. Control groups received $1 \mathrm{ml}$ of $50 \%$ ethanol daily by oral gavage. The study was terminated at the end of 8 weeks of treatment. Hereafter, 20 weeks of age correspond to 0 week of treatment, and 28 weeks of age correspond to 8 weeks of treatment.

\section{Blood pressure measurements}

Blood pressure measurement was carried out on all groups of animals at 0 and 8 weeks of treatment, as described previously. ${ }^{16}$ A CODA multichannel, computerized noninvasive blood pressure system (Kent Scientific, Torrington, CT, USA) with a tail-cuff sphygmomanometer was used to measure systolic blood pressure and an approximate diastolic blood pressure on conscious rats. Briefly, during each measurement cycle, blood will be pushed away from the tail by the volume pressure recording cuff. The occlusion cuff will stop back flow of the blood into the tail. Afterwards, when the occlusion cuff deflates, it will let the blood flow back into the tail and thereby increase the tail volume. The pressure exerted by occlusion cuff during this increase in tail volume is measured as systolic blood pressure, whereas occlusion cuff pressure during the deflation at which the blood flow into and out of the tail equalizes is defined as diastolic blood pressure. ${ }^{17}$

\section{Echocardiography}

Cardiac structure and function were measured in all groups of animals using echocardiography technique at 0 and 8 weeks of treatment; transthoracic twodimensionally guided $\mathrm{M}$-mode and Pulse-Wave Doppler measurements were performed using a Sonos 5500 ultrasound system (Agilent Technologies, Andover, MA, USA) equipped with a $12-\mathrm{MHz}$ (s12) transducer as described by us earlier. ${ }^{7,9,18}$ Two-dimensional M-mode measurements include percentage of left ventricular fractional shortening, left ventricular ejection fraction, cardiac output, left ventricular mass, interventricular septal wall thickness at diastole and systole, left ventricular posterior wall thickness at diastole and systole and left ventricular internal dimensions at diastole and systole. Doppler measurements included isovolumetric relaxation time. Animals were anaesthetized while performing echocardiography.

\section{Electrocardiogram measurements}

Electrocardiogram (ECG) recordings were taken at 0 and 8 weeks of treatment on lightly anesthetized rats using a BioPac MP100 system. The ECG signal was analyzed using Acqknowledge 3.7.3 software (Biopac Systems, BIOPAC Inc., Goleta, CA, USA).

\section{Pressure myography measurements:}

Small Arteries. Rats were sacrificed at 28 weeks of age, and the mesenteric vasculature was isolated. The use of mesenteric arteries was predicated on consideration that (a) mesenteric arteries remodel in human hypertensives; ${ }^{19}$ (b) a large percentage of cardiac output flows through the mesenteric circulation, and therefore it contributes to peripheral resistance; and (3) though coronary, renal, femoral and mesenteric resistance arteries remodel and respond to treatment similarly in rat models of hypertension, ${ }^{20}$ minimal branching in mesenteric small arteries renders them suitable for study by pressure myography. A segment of the mesenteric artery was mounted in a pressure myograph (Living Systems Instrumentation, Burlington, VT, USA) such that vessel walls were parallel without stretch. ${ }^{21}$ To ensure unbiased sampling consistency, all segments were from arterial branches of the third order. Vessels were equilibrated for $1 \mathrm{~h}$ at $37^{\circ} \mathrm{C}$ at $45 \mathrm{~mm} \mathrm{Hg}^{21}$ with aerated Krebs solution ( $\mathrm{pH} \sim 7.4$ ). Vessels were considered viable if $\mathrm{KCl}\left(125 \mathrm{mmoll}^{-1}\right)$ elicited $>50 \%$ constriction.

Vascular mechanics. Vessels were deactivated with $\mathrm{Ca}^{2+}$-free Krebs solution containing $10 \mathrm{mmoll}^{-1}$ EGTA. To obtain pressure-lumen diameter relationships, intraluminal pressure was increased incrementally from 3 to $140 \mathrm{~mm} \mathrm{Hg}$ (10 increments). ${ }^{22}$ Lumen and media dimensions were measured at three points along the length of the vessel for each pressure

Vascular geometry. Lumen and media dimensions were measured at a constant intraluminal pressure of $45 \mathrm{~mm} \mathrm{Hg}{ }^{21}$

Formulas. Media stress, which reflects wall tension in the vessel wall, is calculated as $\sigma=(P D) /(2 \mathrm{WT})$, where $P$ is the intraluminal pressure, and $D$ and WT are the lumen diameter and media thickness, respectively. Pressure is converted as $1 \mathrm{~mm} \mathrm{Hg}=1.334 \times 10^{3} \mathrm{dyn}^{-1}$. $^{2}$

Media strain, which reflects pressure-induced relative change in lumen diameter, is calculated as $\varepsilon=\left(D-D_{0}\right) / D_{0}$, where $D$ is the observed lumen diameter for a given intraluminal pressure and $D_{\mathrm{o}}$ is the baseline diameter measured at $3 \mathrm{~mm} \mathrm{Hg}$.

Elastic modulus (ET) describes the intrinsic elastic properties of the wall material. It is obtained by fitting the stress-strain data from each vessel to an exponential curve $\left(y=a e^{b x}\right): \sigma=\sigma_{\mathrm{o}} e^{\beta \varepsilon}$ where $\sigma_{\mathrm{o}}$ is the stress at the baseline diameter and $\beta$ is a constant related to the rate of increase of the stress-strain curve. Tangential ET is calculated at several values of stress from the derivative of the exponential curve: $\mathrm{ET}=\mathrm{d} \sigma / \mathrm{d} \varepsilon=\beta \sigma_{\mathrm{o}} e^{\beta \varepsilon}$. Intrinsic stiffness of wall components is represented as the slope of the ET vs. stress curve.

Tissue collection. At the end of the study (28 weeks of age), all rats were sacrificed; the heart tissues and other organs were isolated and flash frozen in liquid nitrogen.

Histology: Ventricular tissue was embedded in paraffin. Paraffin-embedded tissue was cut into 7- $\mu \mathrm{m}$ sections on a Microm HM 550 cryostat (Thermo Fisher Scientific Inc., Kalamazoo, MI, USA). The levels of collagen deposition were determined by Masson trichrome staining. Photographs were taken using a Zeiss LSM 5 Pascal microscope (Carl Zeiss MicroImaging, Thornwood, NY, USA).

\section{Oxidative stress measurement}

Lipid peroxidation levels in blood plasma, collected at the time of sacrifice, were measured by estimating the amount of malondialdehyde using the Oxiselect TBARS Assay Kit (Cell Biolabs, San Diego, CA, USA) as described by us earlier ${ }^{8,9}$ by following the manufacturer's instructions. Thiobarbituric acid reactive substance (TBARS) values were expressed as $\mathrm{nmol} \mathrm{ml}^{-1}$ of plasma.

\section{Inflammatory marker measurements}

Serum samples collected at the time of sacrifice were used for biochemical analysis. ELISA kits (Thermo Scientific, IL, USA, USA) were used to measure the serum interleukin- 6 and tumor necrosis factor alpha (TNF- $\alpha$ ) levels. The assays were performed as described earlier ${ }^{23}$ by following the manufacturer's instructions.

\section{Nitrate + nitrite measurements}

Nitrate $\left(\mathrm{NO}_{3}{ }^{-}\right)+$nitrite $\left(\mathrm{NO}_{2}{ }^{-}\right)$were measured in the blood plasma from all the treatment groups using the Nitrate/Nitrite Colorimetric Assay kit from the Cayman Chemical (Ann Arbor, MI, USA) according to the manufacturer's protocol. Before assay, all the samples were thawed and filtered through Amicon $10 \mathrm{kDa}$ molecular-weight cut-off filter (Millipore Corporation, 
Bedford, MA, USA). The readings were taken in a 96-well plate at a wavelength of $540 \mathrm{~nm} . \mathrm{A} \mathrm{NO}_{2}{ }^{-}$standard curve was prepared in each plate to determine total $\mathrm{NO}_{3}{ }^{-} / \mathrm{NO}_{2}{ }^{-}(\mu \mathrm{mol})$

\section{Statistics}

Results are presented as means \pm s.e.m. All the data except pressure myography were analyzed by one-way analysis of variance (ANOVA). Significant values are defined as $P<0.05$. When significance was obtained, ANOVA was followed by Tukey's post hoc test. Pressure myography data were analyzed by one-way or two-way ANOVA for repeated measures, followed as appropriate by StudentNewman-Keuls or Bonferroni post tests to detect between-group differences. $P<0.05$ was considered significant.

\section{RESULTS}

\section{General characteristics of the animal model}

All rats had almost similar increase in the body weight throughout the course of the study. Resveratrol treatment had no effect on the body weight of the animals. However, heart to body weight ratio was significantly higher in 28-week-old SHRs when compared with WKY control rats. The treatment with hydralazine alone or in combination with resveratrol significantly regressed the increase in heart to body weight ratio in SHR. However, we did not observe any reduction in heart to body weight ratio with 8 weeks of resveratrol treatment alone (Table 1).

\section{Blood pressure}

Twenty-week-old SHRs had significantly elevated blood pressure compared with normotensive WKY rats before the treatment started (Supplementary Table 1). Treatment with hydralazine, but not resveratrol, significantly reduced the elevated blood pressure (systolic as well as diastolic) in SHR when compared with untreated group (Table 1). Moreover, hydralazine in conjunction with resveratrol further significantly reduced the systolic blood pressure and diastolic blood pressure in SHR when compared with untreated or hydralazinetreated SHR (Table 1). Please note that volume pressure recording tail-cuff blood pressure system cannot exactly measure the diastolic blood pressure values, unlike systolic blood pressure, because volume pressure recording measurements of diastolic blood pressure have shown consistent variation from the respective telemetry measurements in the validation studies. ${ }^{17}$ Accordingly, the diastolic blood pressure reported in the present study might not be accurate when compared with telemetry.

\section{Cardiac structure}

M-mode echocardiography showed significant increases in interventricular septal wall thickness at diastole and left ventricular posterior wall thickness at diastole in 20-week-old SHRs when compared with age-matched controls before the treatment (Supplementary Table 1). However, hydralazine, but not resveratrol, significantly reduced the increase in interventricular septal wall thickness at diastole and left ventricular posterior wall thickness at diastole in 28-week SHR when compared with untreated group (Table 1). Combination treatment of hydralazine with resveratrol also significantly reduced this increase in SHR (Table 1). SHRs did not exhibit any change in LV internal dimension when compared with WKY controls at 28-week time point (Table 1).

\section{Cardiac function}

A significant prolongation in the diastolic functional parameter, isovolumetric relaxation time, was observed in 20-week SHRs before the treatment in comparison with their respective WKY controls (Supplementary Table 1); and also at 28 weeks of age (Table 2). Hydralazine alone, but not resveratrol, moderately but significantly improved the prolonged isovolumetric relaxation time in SHRs (Table 2). Combination treatment of hydralazine with resveratrol normalized isovolumetric relaxation time in 28-week-old SHRs (Table 2). There was no significant reduction in the systolic functional parameter, fractional shortening in 20-week-old SHR before the treatment (Supplementary Table 1); however at 28 weeks, we observed a significant reduction in fractional shortening in untreated SHR compared with normotensive WKY rats (Table 2). Both resveratrol alone as well as hydralazine alone significantly prevented the drop in fractional shortening in SHR (Table 2); resveratrol in combination with hydralazine was significantly more effective than hydralazine alone in preventing the decrease in fractional shortening (Table 2). Cardiac output was unchanged in all groups at the end of the study (Table 2).

\section{ECG measurements}

The ECG recordings did not show any abnormalities in the QT interval in any of the groups at any time points of the study (Table 2).

\section{Pressure myography of resistance arteries}

Media-to-lumen ratios were greater in SHR vessels compared with WKY rat (Table $3 ; P<0.01$ ). Resveratrol alone had no effect on vascular geometry. However, hydralazine (whether alone or combined

Table 1 Effect of treatment on cardiac hypertrophy, blood pressure and cardiac structure in 28-week-old WKY rats and SHRs treated with resveratrol and/or hydralazine

\begin{tabular}{|c|c|c|c|c|c|c|c|c|}
\hline Variable & $W K Y C$ & WKY H & $W K Y R$ & $W K Y H+R$ & SHR C & SHR H & $S H R R$ & $S H R H+R$ \\
\hline HW/BW (mg per 100g) & $301.87 \pm 6.65$ & $299.75 \pm 5.70$ & $300.42 \pm 6.15$ & $304.38 \pm 4.59$ & $346.38 \pm 2.73 *$ & $324.63 \pm 2.90^{\#}$ & $345.86 \pm 4.43$ & $316 \pm 2.19^{\#}$ \\
\hline $\begin{array}{l}\text { Systolic blood pressure } \\
(\mathrm{mm} \mathrm{Hg})\end{array}$ & $134 \pm 2$ & $139 \pm 3$ & $130 \pm 3$ & $127 \pm 1$ & $213 \pm 3^{*}$ & $162 \pm 4^{\#}$ & $205 \pm 2$ & $141 \pm 2 \#^{€}$ \\
\hline $\begin{array}{l}\text { Diastolic blood pres- } \\
\text { sure }(\mathrm{mm} \mathrm{Hg})\end{array}$ & $83 \pm 5$ & $85 \pm 2$ & $90 \pm 5$ & $85 \pm 2$ & $164 \pm 6^{*}$ & $105 \pm 4^{\#}$ & $154 \pm 5$ & $92 \pm 4 \#^{€}$ \\
\hline IVSd $(\mathrm{cm}) \times 1000$ & $164.5 \pm 5.44$ & $163.75 \pm 4.93$ & $174.57 \pm 4.51$ & $167.29 \pm 3.64$ & $229.67 \pm 8.14^{*}$ & $200.88 \pm 4.24^{\#}$ & $215.63 \pm 7.42$ & $175.13 \pm 2.84 \#^{€}$ \\
\hline LVPWd $(\mathrm{cm}) \times 1000$ & $175.75 \pm 10.24$ & $177 \pm 11.54$ & $167.71 \pm 11.68$ & $171.8 \pm 17.23$ & $213.44 \pm 19.69^{*}$ & $174.63 \pm 8.95^{\#}$ & $196 \pm 10.13$ & $170.14 \pm 9.05^{\#}$ \\
\hline LVIDd (mm) & $422.75 \pm 21.57$ & $434.75 \pm 18.06$ & $417 \pm 18.42$ & $426.83 \pm 20.48$ & $425.89 \pm 11.59$ & $450 \pm 19.08$ & $440.43 \pm 31.28$ & $447.71 \pm 31.51$ \\
\hline
\end{tabular}

Abbreviations: C, control; H, hydralazine; HW/BW, heart weight to body weight ratio; IVSd, interventricular septal wall thickness at diastole; LVIDd, ventricular internal dimensions at diastole; LVPWd, left ventricular posterior wall thickness at diastole; R, resveratrol; SHR, spontaneously hypertensive rat; WKY, Wistar-Kyoto.

Data are mean \pm s.e. $n=8-10$. ${ }^{*} P<0.05$ vs. WKY C; $\# P<0.05$ vs. SHR C; and ${ }^{€} P<0.05$ vs. SHR H. 
with resveratrol) significantly corrected media-to-lumen ratio in SHR vessels $(P<0.05)$.

Vascular compliance is influenced by the fact that transduction of intraluminal pressure to the vessel wall as stress is modulated by the geometry of the artery. Isobaric ET (that is, ET vs. pressure) is determined by two factors-wall component stiffness and vessel geometry. However, when ET is plotted against stress, geometry is mathematically eliminated as a contributor, and therefore provides information regarding solely the stiffness of wall components such as elastin, collagen and smooth muscle cells. Wall component stiffness, presented as the slope of ET vs. stress (Table 3), was increased in SHR vs. WKY vessels $(P<0.01)$. Here, resveratrol treatment alone attenuated stiffening of wall components of SHR arteries, as did hydralazine and the combination of resveratrol with hydralazine $(P<0.01)$.

\section{Histological analysis}

The histological analysis of ventricular tissue showed increased collagen deposition in the area surrounding blood vessels in 28-week-old SHRs compared with its normotensive counterparts, and representative images are shown in Figure 1. Resveratrol alone or in combination with hydralazine was able to reduce the collagen deposition in the perivascular areas of left ventricular tissue from SHR (Figure 1). However, hydralazine alone did not have any effect on the collagen deposition, which remained extensive (Figure 1).

\section{Oxidative stress}

Twenty-eight-week-old SHRs had significantly increased levels of plasma TBARS, when compared with their age-matched control rats; treatment with resveratrol alone or in combination with hydralazine significantly reduced elevated TBARS levels in SHR (Figure 2a).

\section{Inflammatory markers}

Serum interleukin-6 levels were increased in SHR when compared with age-matched WKY rats and were significantly reduced upon resveratrol treatment alone but not with hydralazine alone (Figure 2b). There was a significant increase in the plasma TNF- $\alpha$

Table 2 Effect of treatment on cardiac function in 28-week-old WKY rats and SHRs treated with resveratrol and/or hydralazine

\begin{tabular}{|c|c|c|c|c|c|c|c|c|}
\hline Variable & $W K Y C$ & WKY H & $W K Y R$ & $W K Y H+R$ & SHR C & SHR H & SHR R & $S H R H+R$ \\
\hline IVRt (ms) & $20.5 \pm 0.38$ & $20.13 \pm 0.40$ & $20.29 \pm 0.52$ & $20.71 \pm 0.92$ & $29.78 \pm 0.55^{*}$ & $24.88 \pm 1.20^{€}$ & $28.14 \pm 0.91$ & $22.43 \pm 0.78^{€}$ \\
\hline Fractional shortening (\%) & $47.64 \pm 1.12$ & $47.31 \pm 1.59$ & $48.04 \pm 1.81$ & $47.42 \pm 0.86$ & $37.49 \pm 1.28 *$ & $41.75 \pm 1.58^{€}$ & $45.23 \pm 2.67^{€}$ & $47.39 \pm 1.63^{€ \#}$ \\
\hline Cardiac output (ml min $\left.{ }^{-1}\right)$ & $307.58 \pm 11.31$ & $315.47 \pm 17.40$ & $317.78 \pm 12.12$ & $321.8 \pm 19.81$ & $286 \pm 13.26$ & $312.88 \pm 14.52$ & $300.43 \pm 14.60$ & $339.55 \pm 15.55$ \\
\hline QT interval (ms) & $0.205 \pm 0.003$ & $0.195 \pm 0.006$ & $0.209 \pm 0.003$ & $0.206 \pm 0.002$ & $0.209 \pm 0.003$ & $0.206 \pm 0.002$ & $0.201 \pm 0.003$ & $0.204 \pm 0.005$ \\
\hline
\end{tabular}

Abbreviations: $\mathrm{C}$, control; $\mathrm{H}$, hydralazine; IVRt, isovolumetric relaxation time; R, resveratrol; SHR, spontaneously hypertensive rat; WKY, Wistar-Kyoto.

Data are mean \pm s.e. $n=8-10 .{ }^{*} P<0.05$ vs. WKY $C$; ${ }^{€} P<0.05$ vs. SHR $C$; and ${ }^{\#} P<0.05$ vs. SHR H.

Table 3 Effect of treatment on arterial structure in 28-week-old WKY rats and SHRs treated with resveratrol and/or hydralazine

\begin{tabular}{|c|c|c|c|c|c|c|c|c|}
\hline & WKYC & WKY H & WKY R & $W K Y H+R$ & SHR C & SHR H & $S H R R$ & $S H R H+R$ \\
\hline Media:Iumen at $45 \mathrm{~mm} \mathrm{Hg}$ & $10.57 \pm 0.6$ & $7.79 \pm 0.3$ & $11.16 \pm 0.9$ & $7.59 \pm 0.5$ & $16.56 \pm 1.4^{* *}$ & $13.15 \pm 1.2^{\dagger}$ & $18.29 \pm 1.3^{* *}$ & $13.52 \pm 1.1^{\dagger}$ \\
\hline Slope of elastic modulus vs. stress & $5.34 \pm 0.3$ & $4.19 \pm 0.2$ & $5.57 \pm 0.4$ & $5.32 \pm 0.2$ & $8.16 \pm 1.2$ & $4.57 \pm 0.2^{\ddagger}$ & $5.88 \pm 0.7 \ddagger$ & $4.37 \pm 0.3^{\ddagger}$ \\
\hline
\end{tabular}

Abbreviations: C, control; $\mathrm{H}$, hydralazine; $\mathrm{R}$, resveratrol; SHR, spontaneously hypertensive rat; WKY, Wistar-Kyoto.

Data are mean \pm s.e. $n=6-7 .{ }^{* *} P<0.01$ vs. WKY C; ${ }^{\dagger} P<0.05$ and $¥ P<0.01$ vs. SHR C.
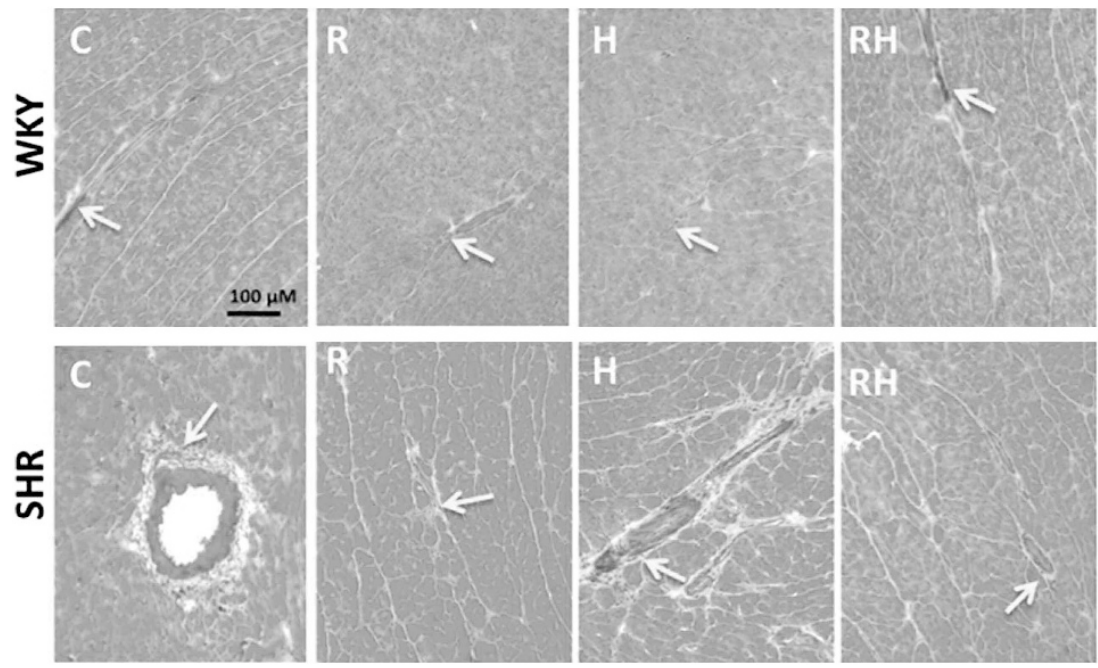

Figure 1 Effect of treatment on myocardial fibrosis in 28-week-old WKY rats and SHRs treated with resveratrol and/or hydralazine. Representative images of WKY or SHR transverse heart ventricular sections from different treatment groups, as indicated, and stained for collagen deposition (Masons trichrome). Fibrotic areas (blue) are indicated by yellow arrows. Muscle stains reddish purple. $\mathrm{C}$, control; $\mathrm{H}$, hydralazine; $\mathrm{R}$, resveratrol; $\mathrm{RH}$, resveratrol plus hydralazine. A full color version of this figure is available at the Hypertension Research journal online. 

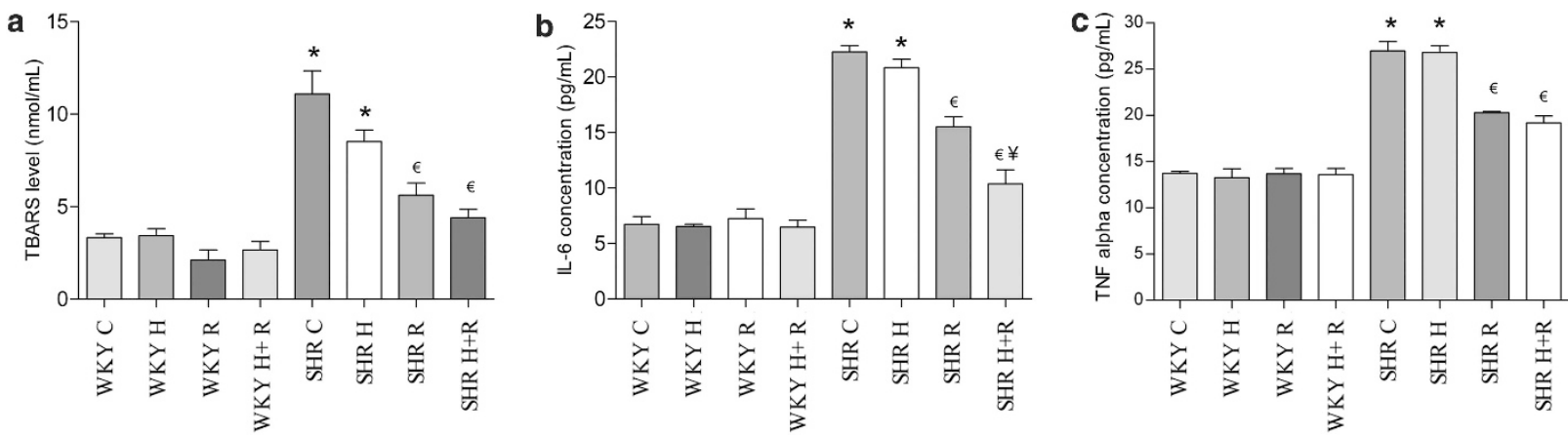

Figure 2 Effect of treatment on oxidative stress and inflammation in 28-week-old WKY rats and SHRs treated with resveratrol and/or hydralazine. (a) Thiobarbituric acid reactive substances (TBARS) (b) Interleukin-6 (IL-6). (c) Tumor necrosis factor alpha (TNF- $\alpha$ ). C, control; H, hydralazine; $\mathrm{R}$, resveratrol. Data are mean \pm s.e. $n=3-5$. ${ }^{*} P<0.05$ vs. WKY $\mathrm{C} ;{ }^{€} P<0.05$ vs. SHR H; and ${ }^{\ddagger} P<0.05$ vs. SHR R.

level in 28-week-old SHRs when compared with their controls (Figure 2c). Resveratrol alone, but not hydralazine, significantly decreased the serum TNF- $\alpha$ level in SHR (Figure 2c). In combination with hydralazine, resveratrol further reduced interleukin- 6 but not TNF- $\alpha$ levels in 28-week-old SHR in comparison with resveratroltreated SHR (Figures $2 \mathrm{~b}$ and $\mathrm{c}$ ).

\section{Nitrate + nitrite levels}

There was a trend toward decreased levels of $\mathrm{NO}_{3}{ }^{-}+\mathrm{NO}_{2}{ }^{-}$in SHRs compared with WKY rats and an improvement with resveratrol hydralazine treatments, but it did not reach statistical significance (Supplementary Table 2).

\section{DISCUSSION}

Although hemodynamic load is a major determinant of cardiac hypertrophy in hypertension, there are blood pressure-independent factors also involved in the pathogenesis and its progression. ${ }^{24}$ Accordingly, prevention or regression of cardiac hypertrophy with different pharmacological agents has had an enormous impact on the prognosis in hypertensive patients. ${ }^{25}$ In this context, we and others had recently reported the prevention of cardiac hypertrophy and its deleterious consequences on heart function in resveratrol-treated young SHRs. ${ }^{8,10}$ Moreover, the anti-hypertrophic effect observed with resveratrol in these studies was independent of the blood pressure-lowering effects, suggesting a direct action of this polyphenol on the cardiac tissue. This is consistent with our recent in vitro study, ${ }^{14}$ where we showed an anti-hypertrophic effect of resveratrol on isolated adult cardiomyocytes exposed to norepinephrine. Earlier studies examined the preventive efficacy of resveratrol in the early stages of hypertension in SHR rather than the reversal of the cardiac abnormalities. In the present study, we focused on whether low-dose resveratrol alone or in a combination with a potent blood pressure-lowering agent can reverse the cardiac impairment due to hypertension after its development. The usage of sub-antihypertensive dose of resveratrol in this study also allowed us to tease out the direct effects of resveratrol on myocardial tissue effects in the absence of blood pressure-lowering effects.

In the current study, we observed that 20-week-old SHR had elevated blood pressure and diastolic dysfunction before the start of the treatment. However at 28 weeks, SHR presented with established hypertension, systolic and diastolic dysfunction without any change in electrical activity. We did not observe a decrease in blood pressure with 8 weeks of resveratrol treatment alone in SHR, but hydralazine treatment alone was able to significantly reduce blood pressure in
SHR. However, when resveratrol was administered in conjunction with hydralazine, it enhanced the antihypertensive action of hydralazine by further decreasing it to near-baseline levels.

We previously reported prevention of cardiac dysfunction in SHR rats with resveratrol; ${ }^{8}$ in the present study, our data show that 8 weeks of treatment with resveratrol was indeed effective in preventing systolic dysfunction but failed to reverse diastolic dysfunction in 28-week-old SHRs. On the other hand, hydralazine was effective in improving both diastolic and systolic function moderately but significantly. However, resveratrol in combination with hydralazine was able to completely restore both systolic and diastolic dysfunction in SHR.

The increased deposition of collagen is a hallmark of the hypertrophic remodeling process and that can predispose to increased risk of adverse cardiac events. ${ }^{26}$ Resveratrol has been reported to inhibit collagen deposition and fibroblast proliferation, two key events in the development of cardiac fibrosis observed in both in vivo and in vitro settings. ${ }^{27,28}$ The reduction in collagen deposition observed in the present study with resveratrol, and resveratrol/hydralazine treatment, but not hydralazine alone, may have contributed to the reduced myocardial stiffness, thereby improving the cardiovascular function in hypertensive rats.

Abnormalities of small arteries are also major contributors to the pathogenesis and maintenance of hypertension. ${ }^{29}$ Changes in the structural and functional properties have been also detected in small arteries from the SHR. In this regard, vascular compliance is determined by passive geometry as well as intrinsic stiffness of arterial wall components. ${ }^{21}$ Thus, the effects of resveratrol on these parameters were considered. The ET vs. stress plot provides information regarding the stiffness of wall components (that is, connective tissue, elastin and collagen fibers, smooth muscle cells and endothelial cells), which is independent of arterial geometry. ${ }^{30} \mathrm{We}$ previously reported that, in the context of developing hypertension (that is, 10-20 weeks of age) in the SHR, the beneficial effects of resveratrol lie in correction of vascular geometry. ${ }^{31}$ In contrast, we observed here in SHR with established hypertension (that is, 20-30 weeks of age) that resveratrol fails to improve vascular geometry. Instead, resveratrol improved the intrinsic stiffness of the arterial wall. Although we did not measure levels of extracellular matrix proteins such as collagen, these findings in the microvasculature are consistent with alleviation of fibrosis that we observed on the heart.

To understand the mechanism underlying the protective effect of resveratrol/hydralazine treatment in reversing the cardiovascular abnormalities in SHR, we examined the status of inflammation and 
oxidative stress, two major contributors in the pathogenesis of hypertensive heart disease. ${ }^{32,33}$ It is well established that oxidative stress and inflammation are inextricably linked to form a circuit in the progression of cardiovascular events, and if not blocked, culminates in progressive target organ injury and dysfunction. ${ }^{34}$ Moreover, previous studies from our laboratory and others have reported that the cardioprotective effect of resveratrol might be attributable to its antioxidant-enhancing activity and/or antiinflammatory activity. ${ }^{8,13,23}$ Consistent with these previous reports, we found that resveratrol alone or in combination with hydralazine was able to reduce the oxidative stress and inflammation in hypertensive rats, which was evident from the TBARS, interleukin-6 and TNF- $\alpha$ measurements. On the other hand, hydralazine treatment alone was able to reduce oxidative stress (TBARS) but not inflammation (interleukin and TNF- $\alpha$ ) in hypertensive rats. These findings imply that stimulation of an inflammatory response elicited the increased perivascular fibrosis observed in SHR rats. This association (between inflammation and fibrosis) is also consistent with the beneficial effect observed in reversing fibrosis with resveratrol, and not with hydralazine, in SHR.

These results are consistent with the previous reports where resveratrol exerted inhibitory effects on central signal transduction pathways and molecules involved with inflammation. These include the arachidonic acid pathway-cyclooxygenase 2 and the activation of the pro-inflmmatory nuclear transcription factor nuclear factor kappa B. ${ }^{35,36}$ Resveratrol can affect immune cell types, including $\mathrm{T}$ and $\mathrm{B}$ cells and macrophages, by reducing reactive oxygen species production and nitric oxide generation by the latter. Resveratrol can also affect pro-inflammatory responses from fibroblastic cells, by preventing cytokine (interleukin-1 $\beta$ )-induced activation of nuclear factor kappa B, and the PI3 kinase pathway, in part via the action of histone deacetylase Sirt $1 .{ }^{37}$

In summary, the improvement observed in the cardiac structure and function with low-dose resveratrol/hydralazine combination therapy might be attributed to either a reduction in the oxidative stress-inflammation axis or a reduction in the hemodynamic load, or both.

\section{CONCLUSION}

Our data shows that 8 weeks of low-dose resveratrol or hydralazine treatment alone was able to alleviate some of cardiovascular defects in hypertensive rats; however, combination therapy of resveratrol with hydralazine had superior effects in reducing blood pressure, improving cardiac structure and function when compared with resveratrol or hydralazine alone. Together, these data provide evidence that the dietary intake of resveratrol (rather than pharmacological dosage) in combination with existing antihypertensive agents may provide optimal outcome in reversing cardiovascular complications in hypertensive patients.

\section{CONFLICT OF INTEREST}

The authors declare no conflict of interest.

\section{ACKNOWLEDGEMENTS}

We would like to acknowledge the RO Burrell laboratory staff at St. Boniface Hospital Research Centre for their great support in conducting animal studies. SJT is the recipient of Manitoba Health Research Council PhD fellowship. XLL is recipient of James Gordon Fletcher PhD fellowship for research in Functional Foods and Nutraceuticals. SZ and HDA are recipients of the New Investigator Award from the CIHR, Canada. TN was the recipient of the Target Obesity New Investigator Award from Heart and Stroke Foundation of Canada and CIHR. This study was supported by operating grants from Heart and Stroke Foundation and Agriculture and Agri-Food Canada held by TN.

1 Roger VL, Go AS, Lloyd-Jones DM, Benjamin EJ, Berry JD, Borden WB, Bravata DM, Dai S, Ford ES, Fox CS, Fullerton HJ, Gillespie C, Hailpern SM, Heit JA, Howard VJ, Kissela BM, Kittner SJ, Lackland DT, Lichtman JH, Lisabeth LD, Makuc DM, Marcus GM, Marelli A, Matchar DB, Moy CS, Mozaffarian D, Mussolino ME, Nichol G, Paynter NP, Soliman EZ, Sorlie PD, Sotoodehnia N, Turan TN, Virani SS, Wong ND, Woo D, Turner MB. Heart disease and stroke statistics-2012 update: a report from the American Heart Association. Circulation 2012; 125: e2-e220.

2 Purushothaman S, Renuka Nair R, Harikrishnan VS, Fernandez AC. Temporal relation of cardiac hypertrophy, oxidative stress, and fatty acid metabolism in spontaneously hypertensive rat. Mol Cell Biochem 2012; 351: 59-64.

3 Sen S, Tarazi RC, Bumpus FM. Cardiac hypertrophy and antihypertensive therapy. Cardiovasc Res 1977; 11: 427-433.

4 Smoliga JM, Baur JA, Hausenblas HA. Resveratrol and health-a comprehensive review of human clinical trials. Mol Nutr Food Res 2011; 55: 1129-1141.

5 Wu JM, Hsieh TC, Wang Z. Cardioprotection by resveratrol: a review of effects/targets in cultured cells and animal tissues. Am J Cardiovasc Dis 2011; 1: 38-47.

6 Yu W, Fu YC, Wang W. Cellular and molecular effects of resveratrol in health and disease. J Cell Biochem 2012; 113: 752-759.

7 Juric D, Wojciechowski P, Das DK, Netticadan T. Prevention of concentric hypertrophy and diastolic impairment in aortic-banded rats treated with resveratrol. Am J Physiol Heart Circ Physiol 2007; 292: H2138-H2143.

8 Thandapilly SJ, Wojciechowski P, Behbahani J, Louis XL, Yu L, Juric D, Kopilas MA, Anderson HD, Netticadan T. Resveratrol prevents the development of pathological cardiac hypertrophy and contractile dysfunction in the SHR without lowering blood pressure. Am J Hypertens 2010; 23: 192-196.

9 Wojciechowski P, Juric D, Louis XL, Thandapilly SJ, Yu L, Taylor C, Netticadan T. Resveratrol arrests and regresses the development of pressure overload- but not volume overload-induced cardiac hypertrophy in rats. J Nutr 2010; 140: 962-968.

10 Dolinsky VW, Chan AY, Robillard Frayne I, Light PE, Des Rosiers C, Dyck JR. Resveratrol prevents the prohypertrophic effects of oxidative stress on LKB1. Circulation 2009; 119: 1643-1652.

11 Liu Z, Song Y, Zhang X, Zhang W, Mao W, Wang W, Cui W, Jia X, Li N, Han C, Liu C. Effects of trans-resveratrol on hypertension-induced cardiac hypertrophy using the partially nephrectomized rat model. Clin Exp Pharmacol Physiol 2005; 32: 1049-1054.

12 Liu ZP, Song Y, Liu ZQ, Zhang XP. [Preventive effect of trans-resveratrol on hypertension-induced cardiac hypertrophy in partially nephrectomized rats]. Wei Sheng Yan Jiu 2005; 34: 756-758.

13 Li HL, Wang AB, Huang Y, Liu DP, Wei C, Williams GM, Zhang CN, Liu G, Liu YQ, Hao DL, Hui RT, Lin M, Liang CC. Isorhapontigenin, a new resveratrol analog, attenuates cardiac hypertrophy via blocking signaling transduction pathways. Free Radic Biol Med 2005; 38: 243-257.

14 Thandapilly SJ, Louis XL, Yang T, Stringer DM, Yu L, Zhang S, Wigle J, Kardami E, Zahradka P, Taylor C, Anderson HD, Netticadan T. Resveratrol prevents norepinephrine induced hypertrophy in adult rat cardiomyocytes, by activating NO-AMPK pathway. Eur J Pharmacol 2011; 668: 217-224.

15 Onaka U, Fujii K, Abe I, Fujishima M. Antihypertensive treatment improves endothelium-dependent hyperpolarization in the mesenteric artery of spontaneously hypertensive rats. Circulation 1998; 98: 175-182.

16 Cipolla MJ, Smith J, Bishop N, Bullinger LV, Godfrey JA. Pregnancy reverses hypertensive remodeling of cerebral arteries. Hypertension 2008; 51: 1052-1057.

17 Feng M, Whitesall S, Zhang Y, Beibel M, D'Alecy L, DiPetrillo K. Validation of volumepressure recording tail-cuff blood pressure measurements. Am J Hypertens 2008; 21 : 1288-1291.

18 Cantor EJ, Babick AP, Vasanji Z, Dhalla NS, Netticadan T. A comparative seria echocardiographic analysis of cardiac structure and function in rats subjected to pressure or volume overload. J Mol Cell Cardiol 2005; 38: 777-786.

19 Short D. Morphology of the intestinal arterioles in chronic human hypertension. Br Heart J 1966; 28: 184-192.

20 Sharifi AM, Li JS, Endemann D, Schiffrin EL. Effects of enalapril and amlodipine on small-artery structure and composition, and on endothelial dysfunction in spontaneously hypertensive rats. J Hypertens 1998; 16: 457-466.

21 Falloon BJ, Bund SJ, Tulip JR, Heagerty AM. In vitro perfusion studies of resistance artery function in genetic hypertension. Hypertension 1993; 22: 486-495.

22 Laurant P, Touyz RM, Schiffrin EL. Effect of pressurization on mechanical properties of mesenteric small arteries from spontaneously hypertensive rats. J Vasc Res 1997; 34: $117-125$.

23 Louis XL, Thandapilly SJ, Mohankumar SK, Yu L, Taylor CG, Zahradka P, Netticadan T. Treatment with low-dose resveratrol reverses cardiac impairment in obese prone but not in obese resistant rats. J Nutr Biochem 2011; 23: 1163-1169.

24 Pachori AS, Numan MT, Ferrario CM, Diz DM, Raizada MK, Katovich MJ. Blood pressure-independent attenuation of cardiac hypertrophy by AT(1)R-AS gene therapy. Hypertension 2002; 39: 969-975.

25 Katholi RE, Couri DM. Left ventricular hypertrophy: major risk factor in patients with hypertension: update and practical clinical applications. Int J Hypertens 2011; 2011. 495349 
26 Grobe JL, Mecca AP, Mao H, Katovich MJ. Chronic angiotensin-(1-7) prevents cardiac fibrosis in DOCA-salt model of hypertension. Am J Physiol Heart Circ Physiol 2006; 290: H2417-H2423.

27 Olson ER, Naugle JE, Zhang X, Bomser JA, Meszaros JG. Inhibition of cardiac fibroblast proliferation and myofibroblast differentiation by resveratrol. Am J Physiol Heart Circ Physiol 2005; 288: H1131-H1138.

28 Wang S, Wang X, Yan J, Xie X, Fan F, Zhou X, Han L, Chen J. Resveratrol inhibits proliferation of cultured rat cardiac fibroblasts: correlated with NO-cGMP signaling pathway. Eur J Pharmacol 2007; 567: 26-35.

29 Mulvany MJ. Small artery remodeling and significance in the development of hypertension. News Physiol Sci 2002; 17: 105-109.

30 Intengan HD, Deng LY, Li JS, Schiffrin EL. Mechanics and composition of human subcutaneous resistance arteries in essential hypertension. Hypertension 1999; 33 (1 Pt 2), 569-574

31 Behbahani J, Thandapilly SJ, Louis XL, Huang Y, Shao Z, Kopilas MA, Wojciechowski P, Netticadan T, Anderson HD. Resveratrol and small artery compliance and remodeling in the spontaneously hypertensive rat. Am J Hypertens 2010; 23: 1273-1278.
32 Pashkow FJ. Oxidative stress and inflammation in heart disease: do antioxidants have a role in treatment and/or prevention? Int J Inflam 2011; 2011: 514623.

33 Vaziri ND, Rodriguez-Iturbe B. Mechanisms of disease: oxidative stress and inflammation in the pathogenesis of hypertension. Nat Clin Pract Nephrol 2006; 2: 582-593.

34 Vaziri ND. Causal link between oxidative stress, inflammation, and hypertension. Iran J Kidney Dis 2008; 2: 1-10.

35 Moreno JJ. Resveratrol modulates arachidonic acid release, prostaglandin synthesis and T6 fibroblast growth. J Pharmacol Exp Ther 2000; 294: 333-338.

36 Zhong LM, Zong Y, Sun L, Guo JZ, Zhang W, He Y, Song R, Wang WM, Xiao CJ, Lu D. Resveratrol inhibits inflammatory responses via the mammalian target of rapamycin signaling pathway in cultured LPS-stimulated microglial cells. PLOS ONE 2012; 7 32195.

37 Busch F, Mobasheri A, Shayan P, Lueders C, Stahlmann R, Shakibaei M. Resveratrol modulates interleukin-1 $\beta$-induced phosphatidylinositol 3-kinase and nuclear factor $\kappa b$ signaling pathways in human tenocytes. J Biol Chem 2012; 287 38050-38063.

Supplementary Information accompanies the paper on Hypertension Research website (http://www.nature.com/hr) 